\title{
Bayesian Shrinkage Estimation of the Relative Abundance of mRNA Transcripts Using SAGE
}

\author{
Jeffrey S. Morris, ${ }^{*}$ Keith A. Baggerly, and Kevin R. Coombes \\ Department of Biostatistics, University of Texas, M. D. Anderson Cancer Center, \\ 1515 Holcombe Blvd., Box 447, Houston, Texas 77030-4009, U.S.A. \\ *email: jeffmo@mdanderson.org
}

\begin{abstract}
Summary. Serial analysis of gene expression (SAGE) is a technology for quantifying gene expression in biological tissue that yields count data that can be modeled by a multinomial distribution with two characteristics: skewness in the relative frequencies and small sample size relative to the dimension. As a result of these characteristics, a given SAGE sample may fail to capture a large number of expressed mRNA species present in the tissue. Empirical estimators of mRNA species' relative abundance effectively ignore these missing species, and as a result tend to overestimate the abundance of the scarce observed species comprising a vast majority of the total. We have developed a new Bayesian estimation procedure that quantifies our prior information about these characteristics, yielding a nonlinear shrinkage estimator with efficiency advantages over the MLE. Our prior is mixture of Dirichlets, whereby species are stochastically partitioned into abundant and scarce classes, each with its own multivariate prior. Simulation studies reveal our estimator has lower integrated mean squared error (IMSE) than the MLE for the SAGE scenarios simulated, and yields relative abundance profiles closer in Euclidean distance to the truth for all samples simulated. We apply our method to a SAGE library of normal colon tissue, and discuss its implications for assessing differential expression.
\end{abstract}

KEY words: Bayesian methods; Bioinformatics; Mixture distributions; Multinomial distribution; SAGE; Shrinkage estimators.

\section{Introduction}

Serial analysis of gene expression (SAGE) is an experimental method for assessing gene expression in a collection of cells. In a typical SAGE experiment, the cells of interest are used to prepare a sample containing large quantities of RNA (about $500 \mu \mathrm{g}$ of total RNA, or about $10^{18}$ mRNA molecules). A prespecified number $n$ of mRNA molecules are selected from the sample, and short sequences (or tags, each 10 nucleotides long) from a unique position within the molecules are sequenced. The number sampled typically lies in the range of $n=10,000$ 100,000 tags. The results of the experiment are recorded as a list of distinct tags with observed frequency counts. See Polyak and Riggins (2001) for a survey article giving more details on SAGE.

Both to illustrate the properties of SAGE data and frame our discussion, we consider a SAGE library of size $n=49,610$ taken from a healthy individual's colon epithelial tissue. This library, referred to as $\mathrm{NC} 1$, is taken from Zhang et al. (1997), and is publically available at http://www.sagenet. org/SAGEData/NC1.htm.

In this sample, 17,703 distinct tags were seen, with observed frequencies ranging from 1 up to a maximum of 1286 . The actual number of unique mRNA transcripts expressed in this tissue is likely larger than 17,703 , since there are probably numerous mRNA species present in the tissue but not observed in our sample. Stollberg et al. (2000) attribute this to sampling error, which they list as one of the four major sources of quantitative error in SAGE. They also developed a method to estimate the number of unique mRNA transcripts expressed, which they find to be $k=25,336$ in this normal colon tissue. Note that the sample size $n$ is of the same order of magnitude as the number of unique tags $k(n / k \approx 2$, here), which is nearly always the case in SAGE experiments.

Upon plotting a histogram of these SAGE counts, it becomes apparent that the distribution of relative frequencies is heavily skewed to the right (see Figure 1). Of the 17,703 distinct tags observed, $13,426(75.8 \%)$ appeared only once in the sample, $1872(10.6 \%)$ twice, and $714(4.0 \%)$ three times. Only $3.6 \%$ of the tags had observed frequencies of nine or greater, yet these accounted for $50 \%$ of the total mRNA mass. Skewness of this type is characteristic of gene expression data (Kuznetsov, 2001).

Since SAGE is an open system, it is useful for characterizing the transcriptome, which entails estimation of the number of unique mRNA transcripts expressed in a given tissue and their relative abundance (e.g., see Velculescu et al., 1999). Estimation of the number of unique tags in the tissue involves estimating the number of species from incomplete multinomial sampling, a very challenging statistical problem (see Bunge and Fitzpatrick (1993) for review). As this is not our 


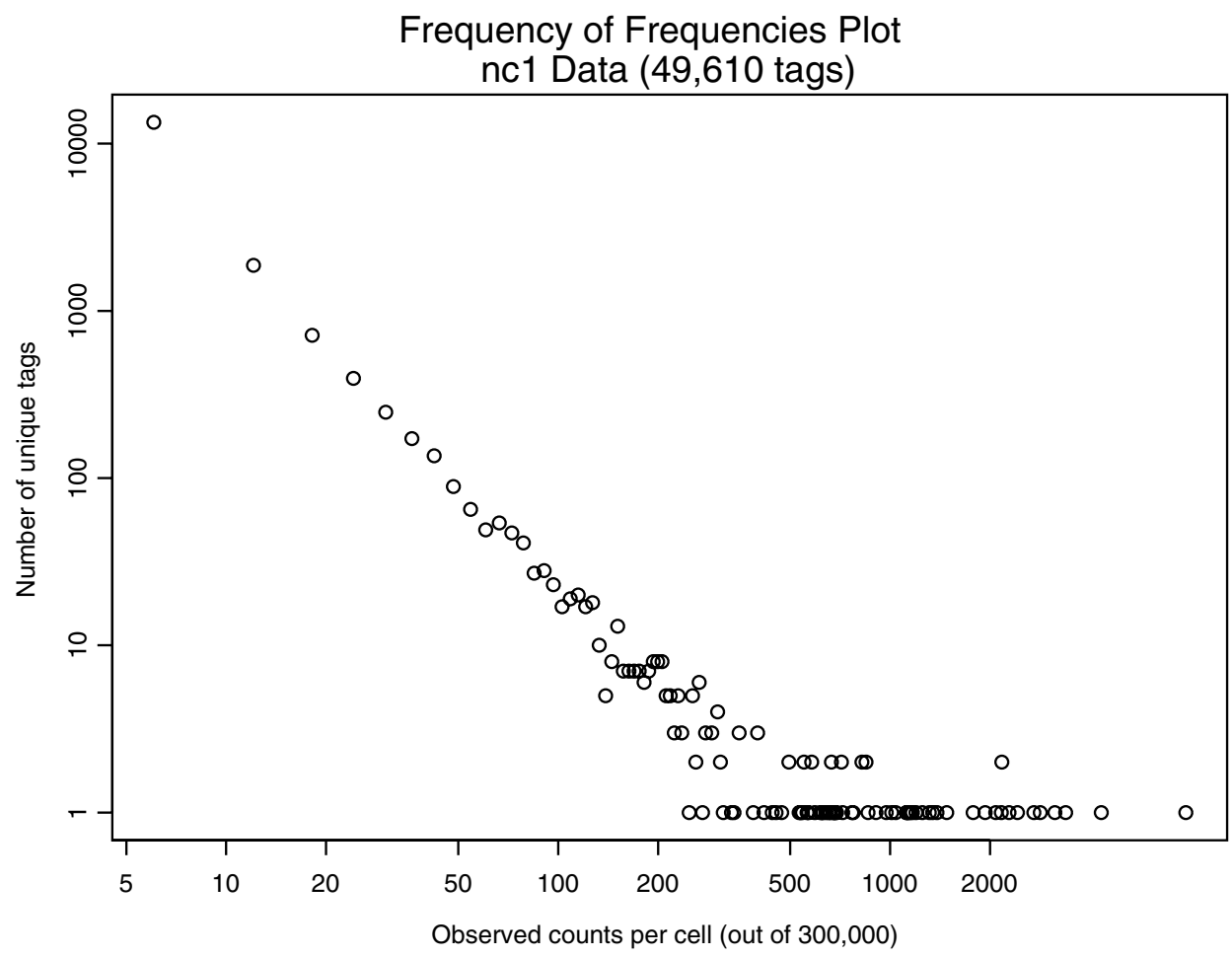

Figure 1. Number of unique tags in SAGE library NC1 with certain observed relative frequencies. Both axes are in the log scale to make the plot more readable. Note the extreme skewness in the distribution of the true frequencies. This linear shape in the log-log scale is characteristic of SAGE data.

focus here, we assume the number of species is known, and investigate the sensitivity of our results to misspecification.

The relative abundance of transcripts is quantified by the relative frequencies of the unique tags. Empirical estimates are easily computed by taking the ratio of the counts for each unique tag and $n$. These estimators ignore the "missing tags," as well as the skewness characteristic of gene expression data, giving them some undesirable properties (see Section 2.1). Our primary focus in this article is to develop a new statistical method that appropriately considers these factors, yielding nonlinear shrinkage estimators that have efficiency advantages over these empirical estimators. Our method is based on a fully specified coherent probability model, so it can be used to perform any desired inferences and is potentially expandable to account for other aspects of SAGE data not modeled in this article.

A common underlying goal of SAGE experiments is identification of tags differentially expressed between two tissue types. We note that estimation of true expression levels necessarily underlies the problem of differential expression, so one may reasonably conjecture that assessments based on more efficient estimators may also have improved sensitivity/ specificity for detecting differentially expressed tags.

One problem with SAGE data is that sequencing errors can occur, which tend to introduce a number of false small count tags that often differ from the true sequence by one or two bases. Before using our method, we assume that a method for detecting and adjusting for these errors (e.g., Colinge and Feger, 2001; Blades, 2002) has been applied to the raw data.
An outline of the remainder of the article is as follows. In Section 2, we propose the multinomial model for SAGE data and demonstrate the weaknesses of some standard estimators. In Section 3, we describe our method, a Bayesian method incorporating a mixture Dirichlet prior. We explain its shrinkage properties and outline how to fit the resulting model using Markov chain Monte Carlo (MCMC). In Section 4, we apply our method to NC1. In Section 5, we describe a simulation study. Section 6 contains discussion of the implications of our results.

\section{Multinomial Model for SAGE Data}

Let $X_{i}$ be the number of occurrences of tag $i$ in our SAGE sample of size $n$, for $i=1, \ldots, k$. Recall $k$ is the total number of expressed tags in the tissue, assumed known. It is natural to model the vector of counts $\underline{X}=\left(X_{1}, \ldots, X_{k}\right)$ as a draw from a multinomial distribution with parameters $n$ and $\underline{\pi}_{k}=\left(\pi_{1}, \pi_{2}, \ldots, \pi_{k}\right)^{T}$, with $\underline{\pi}_{k}$ representing the relative abundance of each tag in the tissue. Since it is estimated that there are 300,000 total mRNA transcripts per cell (Hastie and Bishop, 1976), we report relative frequencies in SAGE as fractions over 300,000, as in Zhang et al. (1997). The joint probability mass function for multinomial vector $\underline{X}$ is $f\left(\underline{X} \mid \underline{\pi}_{k}, n\right)=n ! \prod_{i=1}^{k}\left(\pi_{i}^{X_{i}} / X_{i} !\right)$, with $\sum_{i=1}^{k} X_{i}=n$, $0<\pi_{i}<1 \forall i$ and $\sum_{i=1}^{k} \pi_{i}=1$.

\subsection{Maximum Likelihood Estimation}

The empirical estimators $X_{i} / n$ routinely used in analysis of SAGE data are the MLEs, so they have nice frequentist 
properties, including asymptotic efficiency. However, since $n \sim k$ and $\pi$ is strongly skewed, a typical SAGE library is effectively a small sample, even if $n$ is large. In this setting, the MLE performs well for the relatively few abundant species, but has undesirable properties for the more scarce species comprising the vast majority of the total unique tags. For a given SAGE sample, it underestimates the relative frequencies of all missing tags, and as a result, tends to overestimate the relative frequencies of the scarce observed tags.

Whenever $X_{i}=0$, the corresponding empirical estimator is $\widehat{\pi}_{i, \mathrm{MLE}}=0$, which is outside the parameter space. Thus, for the missing tags, we know that $\widehat{\pi}_{i, \mathrm{MLE}}<\pi_{i}$, so we could say that tag $i$ is underrepresented in the given sample. From the relative frequency constraint $\sum_{i=1}^{k} \pi_{i}=1$, it follows that $\sum_{\left\{i: X_{i}>0\right\}} \widehat{\pi}_{i, \mathrm{MLE}}>\sum_{\left\{i: X_{i}>0\right\}} \pi_{i}$, implying that the observed tags are overrepresented in the given sample on average. Basic multinomial sampling properties suggest more scarce tags are those most likely to be overestimated.

Following is a simple example to illustrate this point. Consider a multinomial population with 51 tags, one abundant tag with a relative frequency of $\pi_{0}=0.50$, and the others all scarce with frequencies $\pi_{i}=0.01$ for $i=1, \ldots, 50$. Consider a hypothetical sample of size 20 from this population. On average, about 40 of the 50 scarce tags will be missing, with the remaining 10 occuring once. Thus, for the scarce tags, we either have an estimator that is out of the parameter space (0) or at best much larger than the true value (0.05). While the MLE is minimum variance unbiased estimator (MVUE), by the sampling characteristics of the problem, the estimator is limited to how well it can estimate the relative frequencies of the scarce tags for a given sample.

The population encountered in SAGE is more complex, but these principles transfer. An estimator that yields positive estimates for the missing tags and shrinks the others in some way may have efficiency advantages over the MLE.

In various multivariate settings, shrinkage estimators have been constructed that uniformly dominate MLEs with respect to IMSE, the mean square error summed over the multivariate parameters (e.g., James and Stein, 1961; George, 1986; Gruber, 1998). These estimators effectively work by taking the MLEs and shrinking them towards a specified prior mean, which can be chosen to obtain efficiency gains in regions of the parameter space that are of particular interest to the investigators. Much of the work involving shrinkage estimation has been done in the multivariate normal setting, and involves linear shrinkage. Shrinkage estimators can be constructed for multinomial data using a Bayesian approach, by specifying a prior distribution for the $\underline{\pi}_{k}$ vector.

\subsection{Bayesian Estimation Using Symmetric Dirichlet Priors}

A standard Bayesian approach for modeling multinomial data is to assume a conjugate Dirichlet prior for the set of probabilities $\underline{\pi}_{k}$, then compute their posterior distribution conditional on the multinomial sample. The joint density for a Dirichlet random variable $\underline{\pi}_{k}$ of dimension $k$ is given by $f\left(\underline{\pi}_{k} \mid \theta_{1}, \theta_{2}, \ldots, \theta_{k}\right)=\left\{\Gamma\left(\sum_{i=1}^{k} \theta_{i}\right) / \prod_{i=1}^{k} \Gamma\left(\theta_{i}\right)\right\} \prod_{i=1}^{k} \pi_{i}^{\theta_{i}-1}$, with $\sum_{i=1}^{k} \pi_{i}=1$.

When there is no prior knowledge of which tags are more likely than others, common practice is to set all Dirichlet parameters to be equal a priori, i.e., $\theta_{i} \equiv \theta$, which we refer to as a symmetric Dirichlet, or $\operatorname{SymmDir}(\theta)$. Under this prior, the posterior mean estimator for each relative frequency is $\widehat{\pi}_{i \text {,Dir }}=\left(X_{i}+\theta\right) /(n+k \theta)$. A common (and, in this case, naive) choice for the hyperparameter is $\theta=1$, described by Jeffreys (1961, Section 3.23), corresponding to a $k$-dimensional generalization of the uniform distribution.

This Bayesian estimator can be viewed as taking the MLEs and shrinking them linearly towards the prior mean, which is $k^{-1}$. The shrinkage is stronger for larger $\theta$, with the estimator being closer to the MLE as $\theta$ approaches 0 . This results in nonzero estimates for all tags, and shrunken estimates for all tags with observed relative frequencies greater than $1 / k$. Applied to our simple example above, a SymmDir(1) prior would yield posterior means of $\widehat{\pi}_{i \text {, Dir }}=1 / 71 \approx 0.014$ whenever $X_{i}=0$, and $\widehat{\pi}_{i \text {, Dir }}=2 / 71 \approx 0.028$ when $X_{i}=1$. These estimates are both closer to the true value 0.01 than the corresponding MLEs. In fact, straightforward calculations show that this estimator has uniformly smaller squared error loss than the MLE for all sparse tags in every multinomial sample of size $n \leq k=51$ taken from the population of our example.

The linear shrinkage inherent in this prior, however, will cause it to perform much worse than the MLE for the abundant tags. In the example above, if the abundant tag is observed $X_{0}=10$ times in a given sample, the posterior mean estimator would be $\widehat{\pi}_{i \text {,Dir }} \approx 0.15$, versus an MLE of $\widehat{\pi}_{i, \mathrm{MLE}}=$ 0.50 . The linear shrinkage to the mean causes values further from $k^{-1}$ to be shrunken the most, inducing extremely large biases that cause it to perform very poorly for more abundant tags.

The method performs poorly because the prior inaccurately represents the characteristics of the population under consideration - it assumes that a priori we think that all unique tags are exchangeable, with relative frequencies of $1 / k$, while in reality they are very heterogeneous. There is an unfortunate connotation of "noninformativeness" that many associate with the uniform distribution, which is inaccurate, since its symmetry implies prior information of homogeneity. This prior belief is effectively strong when $k$ is large relative to $n$, and $\theta$ is not too small.

Thus, we see that the MLEs have disadvantages for scarce tags, and Bayesian methods based on a uniform symmetric Dirichlet prior make inaccurate prior assumptions that lead to poor estimation of the abundant tags. We would like to find an alternative method that obtains improved estimators for the scarce tags without sacrificing so much efficiency on the abundant ones, requiring a nonlinear shrinkage estimator. This method should also ensure the relative frequency constraint $\sum_{i=1}^{k} \pi_{i}=1$ is satisfied.

\section{Bayesian Estimation Using a Mixture Dirichlet Prior}

Recall that the characteristics of gene expression suggest there are a large number of scarce tags and a small number of abundant ones. However, we often do not know a priori what tags will be abundant. We quantify this prior knowledge using a type of mixture Dirichlet prior for the $\underline{\pi}_{k}$. With this prior, the $k$ unique tags are stochastically partitioned between two 
discrete classes representing "abundant" and "scarce" tags, each with their own separate multivariate distributions that are scalar multiples of symmetric Dirichlets.

\subsection{Prior Structure}

In defining our prior, we first introduce a new set of parameters $\left\{\underline{\lambda}, \pi^{*}, \underline{q}\right\}$. Each unique tag, $i=1, \ldots, k$, is assumed to belong to one of two classes, either "abundant" or "scarce," with $\lambda_{i}$ being the indicator of whether tag $i$ belongs to the abundant class. $\underline{\lambda}=\left(\lambda_{1}, \lambda_{2}, \ldots, \lambda_{k}\right)^{T}$ is the vector of such indicators for all tags. Assuming we don't know the identity of the abundant tags a priori, we assume the $\lambda_{i}$ are i.i.d. $\operatorname{Bernoulli}(P)$ random variables, where $P$ is the expected proportion of unique tags belonging to the abundant class.

It is useful to represent the partitioning of the $k$ tags into the two classes by introducing the following notation. The indices of those unique tags belonging to the abundant and scarce classes are given by $\mathcal{A}=\left\{i: \lambda_{i}=1\right\}$ and $\mathcal{S}=\left\{i: \lambda_{i}=\right.$ $0\}$, respectively, which are of length $k_{A}=\sum_{i=1}^{k} \lambda_{i}$ and $k_{S}=$ $\sum_{i=1}^{k}\left(1-\lambda_{i}\right) \cdot \pi^{*}=\sum_{i \in \mathcal{A}} \pi_{i}$ represents the "abundant mass," or the total mass of the tags belonging to the abundant class. Likewise $1-\pi^{*}$ represents the "scarce mass." $\pi^{*}$ is given a $\operatorname{Beta}\left(\alpha_{\pi^{*}}, \beta_{\pi^{*}}\right)$ prior.

The vector $q$ of dimension $k$ contains the relative frequencies for each tag within its class. Given the class membership parameters $\underline{\lambda}, \underline{q}$ is partitioned into $\underline{q}_{\mathcal{A}}=\underline{\pi}_{\mathcal{A}} / \pi^{*}$ and $\underline{q}_{\mathcal{S}}=\underline{\pi}_{\mathcal{S}} /\left(1-\pi^{*}\right)$, which are given separate symmetric Dirichlet priors with parameters $\theta_{A}$ and $\theta_{S}$, respectively. Note that the interpretation of $q_{i}$ depends on the class to which tag $i$ belongs. If abundant $\left(\lambda_{i}=1\right)$, then $q_{i}$ represents the proportion of abundant mass attributable to tag $i$, while if scarce $\left(\lambda_{i}=0\right)$, then $q_{i}$ is the proportion of scarce mass attributable to tag $i$. This construct allows the two classes to have separate multivariate distributions, yet retains the relative frequency constraint $\sum_{i=1}^{k} \pi_{i}=1$.

Following is a summary of our mixture Dirichlet prior structure. $\underline{q}_{\mathcal{A}} \mid \underline{\lambda}=$ symmetric $\operatorname{Dirichlet}\left(\theta_{A}\right), \underline{q}_{\mathcal{S}} \mid \underline{\lambda}=$ symmetric $\operatorname{Dirichlet}\left(\theta_{S}\right), \lambda_{i}=$ i.i.d. Bernoulli $(P), \pi^{*}=\operatorname{Beta}\left(\alpha_{\pi^{*}}, \beta_{\pi^{*}}\right)$. The relative frequencies for the unique tags are easily constructed using these quantities as follows: $\pi_{i}=$ $\left\{q_{i} \pi^{*}\right\}^{\lambda_{i}}\left\{q_{i}\left(1-\pi^{*}\right)\right\}^{\left(1-\lambda_{i}\right)}$.

\subsection{The Rich, the Poor, and Robin Hood}

We now present an analogy to further illuminate the heuristics behind this problem and explain why we believe nonlinear shrinkage estimators are appropriate. For tags with zero counts, the standard empirical estimates (MLEs) give zero relative frequency estimates, which are outside the parameter space. Given we know they exist, we would like to give some positive probability to these tags, but because of the relative frequency constraint that the total probability mass must sum to one, this requires decreasing some the estimates for the other observed tags. There's no free lunch-in order to "pay" the zero count tags, we need to "steal" some probability mass from other tags.

A Bayesian estimator does this. The posterior mean can be written as a weighted average of the MLE and prior mean, and thus it effectively shrinks the MLEs towards the prior mean.
In this way, we obtain positive estimates for the zero counts and subsequently shrink the estimates for the observed tags towards their prior mean. The choice of prior determines the shrinkage rule, i.e., from whom we "steal" in order to "pay the zeros."

By performing linear shrinkage, the simple symmetric Dirichlet prior is what we could call a "Robin Hood" prior. That is, it steals the most mass from the "richest" or most abundant tags. This makes sense if we actually believe that the tags are exchangeable, since then large counts are taken to be aberrations for which the most reasonable course of action is to shrink them the most. However, in settings where the exchangeable hypothesis is not true, such as with SAGE, this type of shrinkage is undesirable, leading to estimators with poor properties.

What we would like in our setting is a reverse Robin Hood prior. We would like to steal from the most poor tags (with low counts) and leave the rich alone, since the sampling properties of the problem suggest the poorer tags are the ones holding on to the mass rightfully belonging to the zero tags. In other words, we would like an estimator that shrinks the MLEs nonlinearly, wherein tags with large counts are left largely unaffected, but those with small counts shrunken. This can be accomplished using our mixture Dirichlet prior.

\subsection{Nonlinear Shrinkage and the Mixture Dirichlet Prior}

We now describe how our mixture Dirichlet prior yields nonlinear shrinkage profile. For the following illustration, suppose we know the values of $P, \pi^{*}$, and $\underline{\lambda}$. In this case, the posterior mean relative frequency estimators for scarce tag $i$ and abundant tag $i^{\prime}$ can be written in closed form as

$$
\begin{gathered}
\widehat{\pi}_{i, \text { strat }}=\left(1-\pi^{*}\right)\left(\frac{X_{i}+\theta_{S}}{n_{S}+k_{S} \theta_{S}}\right), \\
\widehat{\pi}_{i^{\prime}, \text { strat }}=\pi^{*}\left(\frac{X_{i^{\prime}}+\theta_{A}}{n_{A}+k_{A} \theta_{A}}\right),
\end{gathered}
$$

where $k_{A}$ and $k_{S}$ are the number of abundant and scarce tags, respectively, and $n_{A}=\sum_{i \in \mathcal{A}} X_{i}$ and $n_{S}=\sum_{i \in \mathcal{S}} X_{i}$ are the total observed counts for tags in the abundant and scarce classes.

Making use of the approximations $n_{S} / n \approx 1-\pi^{*}$ and $k_{S} / k \approx 1-P$, we can simplify (1) to be written as a linear combination of the MLE, $\widehat{\pi}_{i, \mathrm{MLE}}=X_{i} / n$, and the prior mean for a scarce tag, $\pi_{S}=\left\{\left(1-\pi^{*}\right) /(1-P)\right\} *(1 / k)$, with the weight placed on the MLE being $\gamma_{S}=n /\left\{n+\left(1-\pi^{*}\right)^{-1}\right.$ $\left.\times(1-P) k \theta_{S}\right\}$. This weight is typically $<0.5$ under the conditions of SAGE, with $n \sim k, \pi^{*}$ large, and $P$ small. For example, if $\pi^{*}=0.50, P=0.01, \theta_{S}=1$, and $n / k=1$, we get $\gamma_{S}=0.33$. As a result, scarce tags are shrunken heavily towards their prior mean $\pi_{S}$, which is very close to zero. This shrinkage towards the mean causes species with zero counts to have positive relative frequency estimators, and causes tags with counts greater than $n * \pi_{S}$ to be shrunken.

Similarly, we can simplify (2) to be written approximately as a linear combination of the MLE and the prior mean for an abundant tag, $\pi_{A}=\left(\pi^{*} / P\right)(1 / k)$, with the weight on the MLE being $\gamma_{A}=n /\left\{n+\left(\pi^{*}\right)^{-1}(P) k \theta_{A}\right\}$, which is $\approx 1$ under 
the conditions of our problem. Using the example above, with $\theta_{A}=0.5$, we get $\gamma_{A}=0.99$. As a result, abundant tags are shrunken towards their prior mean $\pi_{A}$, but since $\gamma_{A} \approx 1$, the magnitude of this shrinkage is very weak, thus resulting in estimates close to the MLEs, as desired.

The estimates given above assume knowledge of $\underline{\lambda}$ and $\pi^{*}$, which we, of course, do not have. These approximate results hold for tags whose membership in either the scarce or abundant class, given its observed count, is unquestionable, i.e., whose posterior probability of $\lambda_{i}=1$ is close to zero or one. For tags in between, the estimator is effectively a combination of (1) and (2), with the weight placed on each depending on the posterior probability that $\lambda_{i}=1$. This can be viewed as Bayesian model averaging over models with tag $i$ belonging to either the abundant or scarce class, which yields a smooth nonlinear shrinkage profile that shrinks less for larger observed counts. Figure 2 plots the symmetric and mixture Dirichlet estimators versus the observed counts, with the solid line indicating the unshrunken MLEs; it clearly demonstrates the linear and nonlinear shrinkage profiles.

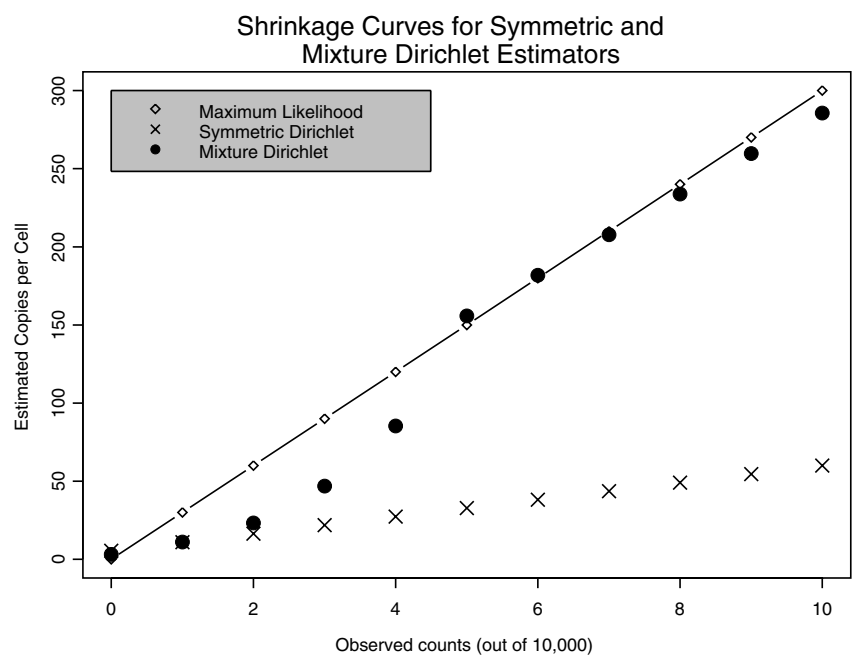

Figure 2. Shrinkage curves for Bayesian estimators using our mixture Dirichlet prior and a symmetric Dirichlet prior for SAGE data with $n=10,000$ and $k=44,984$. The shrinkage curves plot the Bayesian estimates versus the observed counts to demonstrate their shrinkage profiles relative to the MLE, indicated by the solid line. The hyperparameters of the mixture Dirichlet are $\theta_{S}=1.0$ and $\theta_{A}=0.5$, with $P=0.0425$, and a uniform priors for $\pi^{*}$. The hyperparameter for the symmetric Dirichlet distribution is $\theta=1.0$. Note the nonlinear shrinkage profile for the mixture Dirichlet contrasted with the linear profile for the simple symmetric Dirichlet. This plot only gives the shrinkage plot for observed counts from 0 to 10 . If it were extended to include larger counts, the mixture Dirichlet would remain relatively close to the MLE line, while the symmetric Dirichlet would continue on its linear course, moving further and further away from the MLE.

\subsection{Selection of Prior Hyperparameters}

The prior hyperparameters $\theta_{A}, \theta_{S}$, and $P$ work together to determine the shape of the shrinkage curve. We now discuss their effect, and give recommendations for their selection.

Generally, larger values for $\theta_{A}$ and $\theta_{S}$ tend to result in stronger shrinkage towards the prior mean within the respective classes. Since we would like to have little shrinkage for the abundant tags, a natural thought would be to make $\theta_{A}$ very close to zero. However, choosing $\theta_{A}$ too small relative to $\theta_{S}$ will make the boundary between scarce and abundant species very sharp, resulting in a nonsmooth shrinkage profile. This increases the mean squared error (MSE) for tags near the boundaries of the scarce and abundant classes (the intermediate tags described in Section 5). Thus, our general recommendation is to choose $\theta_{A}<\theta_{S}$ to reduce the amount of shrinkage in the abundant tags, but with $\theta_{A}$ not too close to zero.

The hyperparameter $P$ represents the expected relative size of the abundant class. We have found that the model is sensitive to this parameter, with degenerate mixtures resulting if $P$ is chosen to be too small or too large (see Figure 5). To avoid this problem, we propose an automatic diagnostic for the computational procedure that adjusts $P$ if it is clear that it is leading to a degenerate mixture. It is based on two assumptions: 1$)$ The tag with the highest count $\left(X_{\max }\right)$ should be abundant with probability one. 2) Any tag with count 1 should be scarce with large probability (at least $\xi$, e.g., $0.50)$. As the chain proceeds, if we find that at any iteration $\operatorname{Pr}\left(\lambda_{i}=1 \mid X_{i}=X_{\max }\right)<1-\epsilon$ for some small $\epsilon$, then replace $P$ with $P+\delta$ for some $\delta$ and restart the chain, since it is clear that with $P$, the chain was moving towards the degenerate state of all tags being scarce. If $\operatorname{Pr}\left(\lambda_{i}=1 \mid X_{i}=1\right)>$ $1-\xi$, then replace $P$ with $P-\delta$ and restart the chain to avoid the degenerate case of all tags being abundant. Both conditions should not hold simultaneously, but it is possible to oscillate between them, indicating that one should decrease $\delta$.

Given $P$, we have found that a $\operatorname{Uniform}(0,1)$ prior works well for $\pi^{*}$.

\subsection{Fitting the Model}

The posterior distribution of the $\pi_{k}$ under the stratified Dirichlet prior structure is not available in closed form as long as $\underline{\lambda}$ is unknown, so we use a Gibbs sampler for estimation. Following are the steps of the Gibbs sampler, in order.

1. Sample $\underline{\lambda}$ from $f(\underline{\lambda} \mid \underline{X}, P)$, described below. Based on this sample, redefine the indices $\mathcal{A}=\left\{i: \lambda_{i}=1\right\}$ and $\mathcal{S}=$ $\left\{i: \lambda_{i}=0\right\}$.

2. Sample $\pi^{*}$ from its full conditional, which is $\operatorname{Beta}\left(\alpha_{\pi^{*}}+n_{A}, \beta_{\pi^{*}}+n_{S}\right)$.

3. Sample $\underline{q}_{A}$ and $\underline{q}_{S}$ from their full conditionals, which are Dirichlets of dimension $k_{A}$ and $k_{S}$ with parameters $\left\{X_{i}+\right.$ $\left.\theta_{A}, i \in \mathcal{A}\right\}$ and $\left\{X_{i}+\theta_{S}, i \in \mathcal{S}\right\}$, respectively.

In step 1 , the $\lambda_{i}$ can be updated one at a time by drawing $u \sim$ Uniform $(0,1)$, and setting $\lambda_{i}=1$ if $u<\alpha_{i}$, with the $\alpha_{i}=$ $\operatorname{Pr}\left(\lambda_{i}=1 \mid \underline{\lambda}_{(-i)}, \underline{X}, P\right)$, where $\underline{\lambda}_{(-i)}$ is the set of all $\underline{\lambda}$ except $\lambda_{i}$. The expression for $\alpha_{i}$ can be written as $O_{i} /\left(O_{i}+1\right)$, where $O_{i}$ is the conditional posterior odds that tag $i$ is abundant; it 
is the product of the prior odds $P /(1-P)$ and the conditional Bayes factor $\mathrm{BF}_{i}$, given by

$$
\begin{aligned}
\mathrm{BF}_{i}= & \left\{\frac{\Gamma\left(n_{A(-i)}+\alpha_{\pi^{*}}\right) \Gamma\left(n_{S(-i)}+\beta_{\pi^{*}}\right)}{\Gamma\left(n+\alpha_{\pi^{*}}+\beta \pi^{*}\right)}\right\} \times\left\{\frac{\Gamma\left(\theta_{A}+X_{i}\right) \Gamma\left(\theta_{S}\right)}{\Gamma\left(\theta_{A}\right) \Gamma\left(\theta_{S}+X_{i}\right)}\right\} \\
& \times\left\{\frac{\left(n_{A(-i)}+X_{i}\right) ! n_{S(-i)} !}{n_{A(-i)} !\left(n_{S(-i)}+X_{i}\right) !}\right\} \\
& \times\left[\frac{\Gamma\left(\theta_{A} k_{A(-i)}+n_{A(-i)}\right) \Gamma\left(\theta_{A} k_{A(-i)}+\theta_{A}\right)}{\Gamma\left(\theta_{A} k_{A(-i)}\right) \Gamma\left(\theta_{A} k_{A(-i)}+\theta_{A}+n_{A(-i)}+X_{i}\right)}\right] \\
& \times\left[\frac{\Gamma\left(\theta_{S} k_{S(-i)}+\theta_{S}+n_{S(-i)}+X_{i}\right) \Gamma\left(\theta_{S} k_{S(-i)}\right)}{\Gamma\left(\theta_{S} k_{S(-i)}+\theta_{S}\right) \Gamma\left(\theta_{S} k_{S(-i)}+n_{S(-i)}\right)}\right] .
\end{aligned}
$$

$\Gamma(x)=\int_{0}^{\infty} \exp (-u) u^{x-1} d u$ is the Gamma function, $k_{A(-i)}=$ $\sum_{j \neq i} \lambda_{j}$ and $k_{S(-i)}=\sum_{j \neq i}\left(1-\lambda_{j}\right)$ are the number of abundant and scarce tags, leaving out transcript $i$, and $n_{A(-i)}=$ $\sum_{j \neq i} \lambda_{j} X_{j}$ and $n_{S(-i)}=\sum_{j \neq i}\left(1-\lambda_{j}\right) X_{j}$ are the total abundant and scarce counts, again leaving out transcript $i$. If one is willing to impose monotonicity (if $\lambda_{i}=1$, then $\lambda_{i^{\prime}}=1$ for all $i^{\prime}: X_{i^{\prime}}>X_{i}$ ), then the parameter $k_{S}$ is sufficient for the $\underline{\lambda}$, and samples from its posterior can be obtained using a single Metropolis step.

For illustration, with $n=10,000, \theta_{S}=1$, and $\theta_{A}=0.5$, $P \approx 0.01$ and $\pi^{*} \approx 0.40$, when $X_{i}=1,2$, and 3 , we have $\alpha_{i} \approx 0.007,0.03$, and 0.12 , respectively. When $X_{i}>9$, we see $\alpha_{i}>0.995$. Whenever $X_{i}=0$, we set $\lambda_{i}=0$ with probability 1 , which saves considerable computation time. The $\mathrm{C}++$ code for performing the MCMC and the derivations for all the conditional distributions given above are available by request from the first author.

\section{Example: Application to NC1}

We now apply our method to $\mathrm{NC} 1$ to estimate the relative abundance of the expressed transcripts in this individual's normal colonic tissue. We start by assuming the number of unique expressed mRNA transcripts is $k=25,336$, as estimated for this tissue by Stollberg et al. (2000). Our simulation studies in the next section suggest that our method's performance is robust with respect to $k$, so this estimate seems sufficient. Before fitting our model, we correct for likely sequencing errors using the method of Blades (2002), yielding 17,264 unique tags, with a maximum revised count of 1314 . The Dirichlet parameters used were $\theta_{S}=1.0$ and $\theta_{A}=0.2$, and $\pi^{*}$ was given a uniform prior. $P$ was chosen to be 0.005 . The results presented here are from a single chain of 2000 Gibbs samples after a burn-in of 500 .

Our method gives joint posterior samples for the relative frequencies of all unique tags, but for this article, we simply demonstrate how they compare with other estimators by plotting the shrinkage curve in Figure 3. The relative frequency estimates from our method are generally smaller than the MLE for tags with observed counts less than 5, and very close to the MLE for more abundant tags. The arguments made in Section 2 suggest that our estimators should have efficiency benefits over the other two methods, which we now investigate using a simulation study.

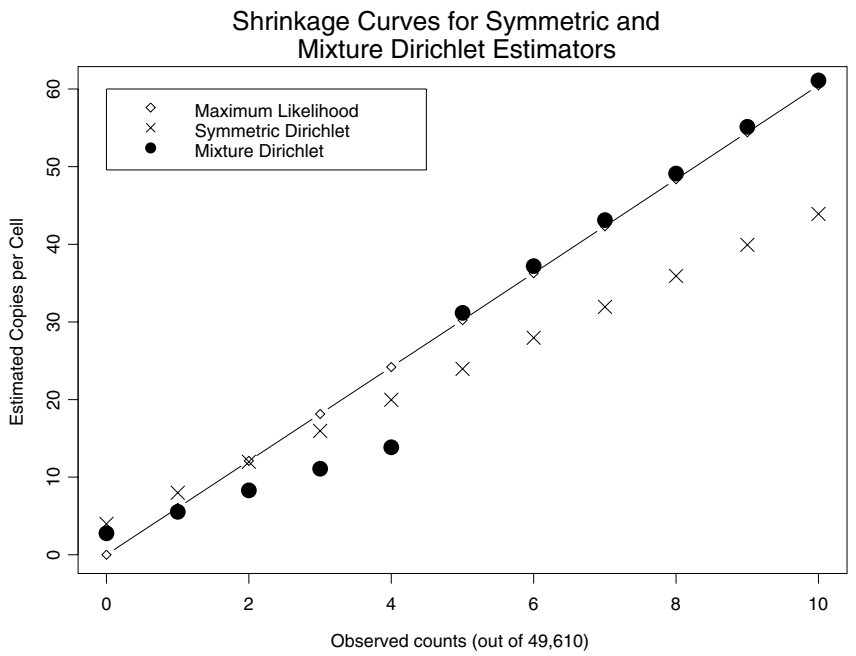

Figure 3. Shrinkage curves for mixture Dirichlet estimator applied to the NC1 data set, assuming $k=25,536$ unique transcripts. The reference line corresponds to the MLEs. The hyperparameters for the mixture Dirichlet are $\theta_{S}=1.0$ and $\theta_{A}=0.2$, with $P=0.005$ and uniform prior on $\pi^{*}$.

\section{Simulation Study}

The "true" set of relative frequencies $\underline{\pi}_{k}$ for simulation were obtained by pooling together the observed counts from six SAGE libraries from breast cancer tissue in a study at M. D. Anderson Cancer Center. These libraries contain a total of 495,947 sequenced tags, with $k=44,984$ unique tags. Of the unique tags, 684 (1.5\%) have observed relative frequencies of greater than 50 copies per cell (i.e., $\pi_{i}>50 / 300,000$ ), accounting for $41 \%$ of the total mRNA mass. Given the large number of sequenced tags, the observed relative frequencies in this pooled sample should give a reasonable approximation of the distribution of true relative frequencies for unique tags in a biological tissue sample (see Velculescu et al., 1999).

We performed simulations based on sample sizes of $n=10,000$ and $n=50,000$, currently among the most prevalent sample sizes seen in the SAGE literature. In each case, we randomly generated 100 samples of size $n$ from a multinomial population with relative frequencies $\underline{\pi}_{k}$. For each sample, we obtained estimates using four methods, maximum likelihood, Bayesian with symmetric Dirichlet(1) prior, Bayesian with our mixture Dirichlet prior, and empirical Bayes estimates using a method described by Good (1953) and attributed to Turing. This estimator is empirical Bayes and involves substituting a smoothed histogram of the observed counts for the histogram of the true counts. For the mixture method, we used $\theta_{S}=1.00$ and $\theta_{A}=0.50$ for the Dirichlet parameters and a uniform prior for $\pi^{*}$. The parameter $P$ was chosen to yield a nondegenerate shrinkage profile. To assess sensitivity to estimation of $k$, the $n=10,000$ simulations were run with estimates of $k$ smaller $(25,000)$ and larger $(65,000)$ than the true value $(44,984)$, and various values of $P(0.01,0.03$, $0.0425,0.06)$ were chosen to assess sensitivity to $P$. Our results for each simulated dataset come from a single chain of Gibbs samples, 2000 for $n=10,000$ simulations and 500 for $n=50,000$ simulations, after a burn-in of 100 . 

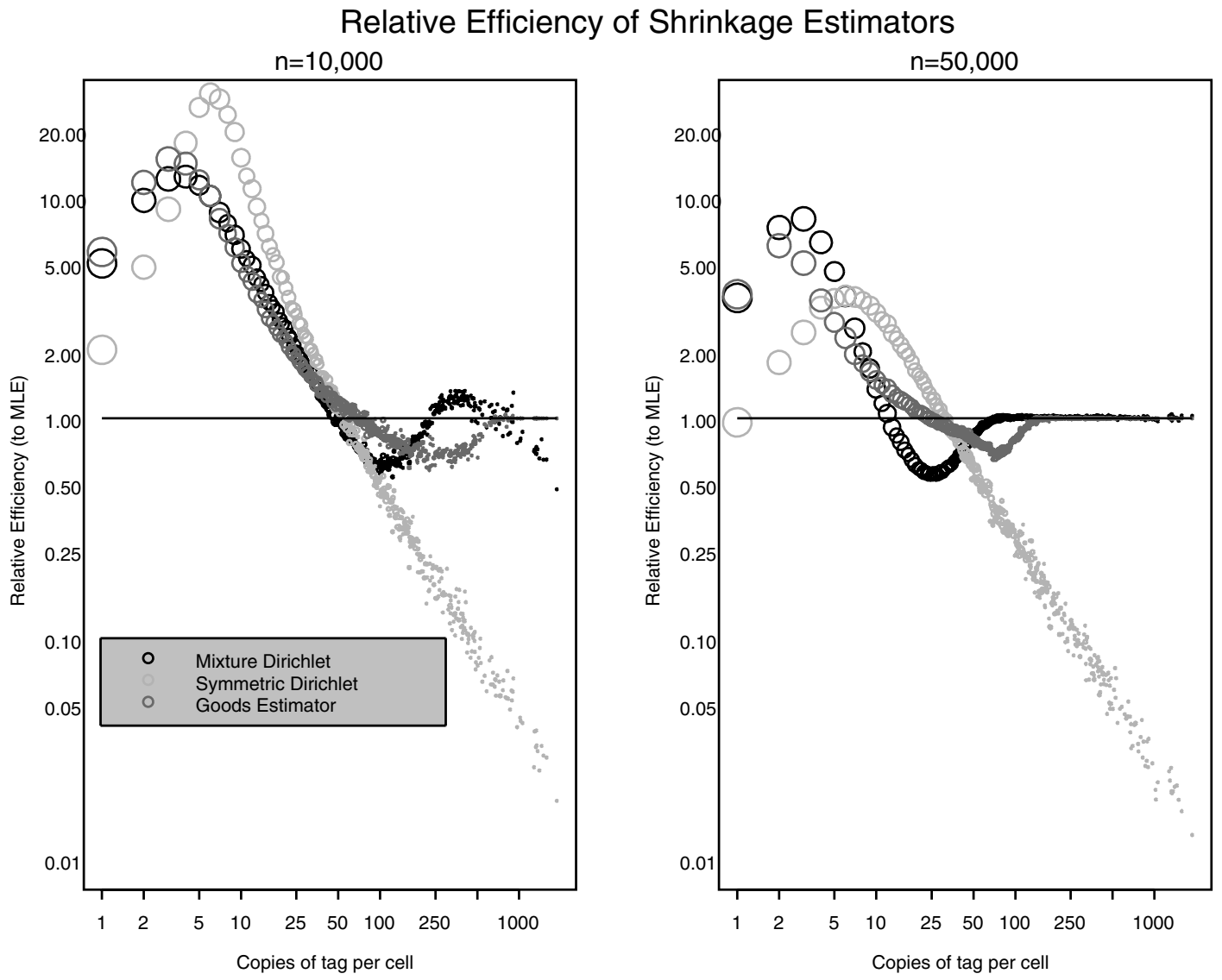

Figure 4. Relative efficiency of estimators from a simulation of 100 multinomial samples of size 10,000 and 50,000 taken from a SAGE-like population. The horizontal axis consists of true relative frequencies multiplied by 300,000 to represent number of copies per cell containing 300,000 total mRNA transcripts. To aid presentation, the results for unique tags with like true relative frequencies have been combined, and the size of each plotted circle made proportional to log(number of unique tags) with that true relative frequency.

The squared error loss for the three estimators was calculated for each of the 44,984 tags in each dataset. The squared error loss for an estimator for tag $i$ in dataset $j$ is $\mathrm{SE}_{i j}=\left(\widehat{\pi}_{i j}-\pi_{i}\right)^{2}$. From this, the mean square error for each tag $\mathrm{MSE}_{i}=100^{-1} \sum_{j=1}^{100} \mathrm{SE}_{i j}$ was computed, as was the relative efficiency $\left(\mathrm{RE}_{i}\right)$ to the MLE. Summing $\mathrm{MSE}_{i}$ over tags, we also computed the integrated mean squared error, IMSE = $\sum_{i=1}^{k} \mathrm{MSE}_{i}$, for each estimator. To compare estimators on a dataset-by-dataset basis, we computed the integrated squared error for each sample $j$, given by $\operatorname{ISE}_{j}=\sum_{i=1}^{k} \mathrm{SE}_{i j}$.

Figure 4 contains plots of the relative efficiency of the symmetric and mixture Dirichlet estimators as a function of the true relative frequencies in the two simulations. First, consider the performance of the symmetric Dirichlet. For both sample sizes, the estimator was more efficient than the MLE for scarce tags, but performed increasingly poorly for more abundant tags, with the relative efficiency close to zero for the most abundant ones. The IMSE for $n=10,000$ and $n=50,000$ were 4489 and 1546, respectively, versus 995 and 601 for the MLE. This was what we expected, per our discussion in Section 2.2.

For $n=10,000$, the mixture Dirichlet estimator showed efficiency improvements of more than $35 \%$ over the MLE, based on IMSE (IMSE = 995 for MLE vs. 643 for mixture Dirichlet). Efficiency gains of this order were seen for every one of the 100 simulated datasets, as measured by ISE. It is important that we do not limit ourselves to aggregate performance measures in this setting, but also examine performance across the various regions of the parameter space, since a loss function treating all tags equally may not match the inferential goals of a given investigation. For the scarce tags (0-50 copies per cell), the mixture method was more efficient with $\mathrm{RE}$ of up to 12 . These scarce tags account for $98.4 \%$ of the total number of unique tags. In the region of $200-1000$ copies per cell $(0.37 \%$ of total tags), its performance was essentially equivalent to the MLE. For an intermediate range (50-200 copies per cell, $1.2 \%$ of tags) and for the most abundant tags ( $>1000$ copies per cell, $0.03 \%$ of tags), the mixture method was outperformed by the MLE, with minimum RE near 0.60 in the intermediate range and 0.50 for the most abundant tag in the population. Turing's estimator had identical IMSE to the mixture Dirichlet (643), with slightly extended regions of improved efficiency (0-60 copies per cell) and reduced efficiency (60-500 copies per cell) within the parameter space.

Figure 5 contains the relative efficiency and shrinkage plots for various choices of $P$. If chosen too small, the model 

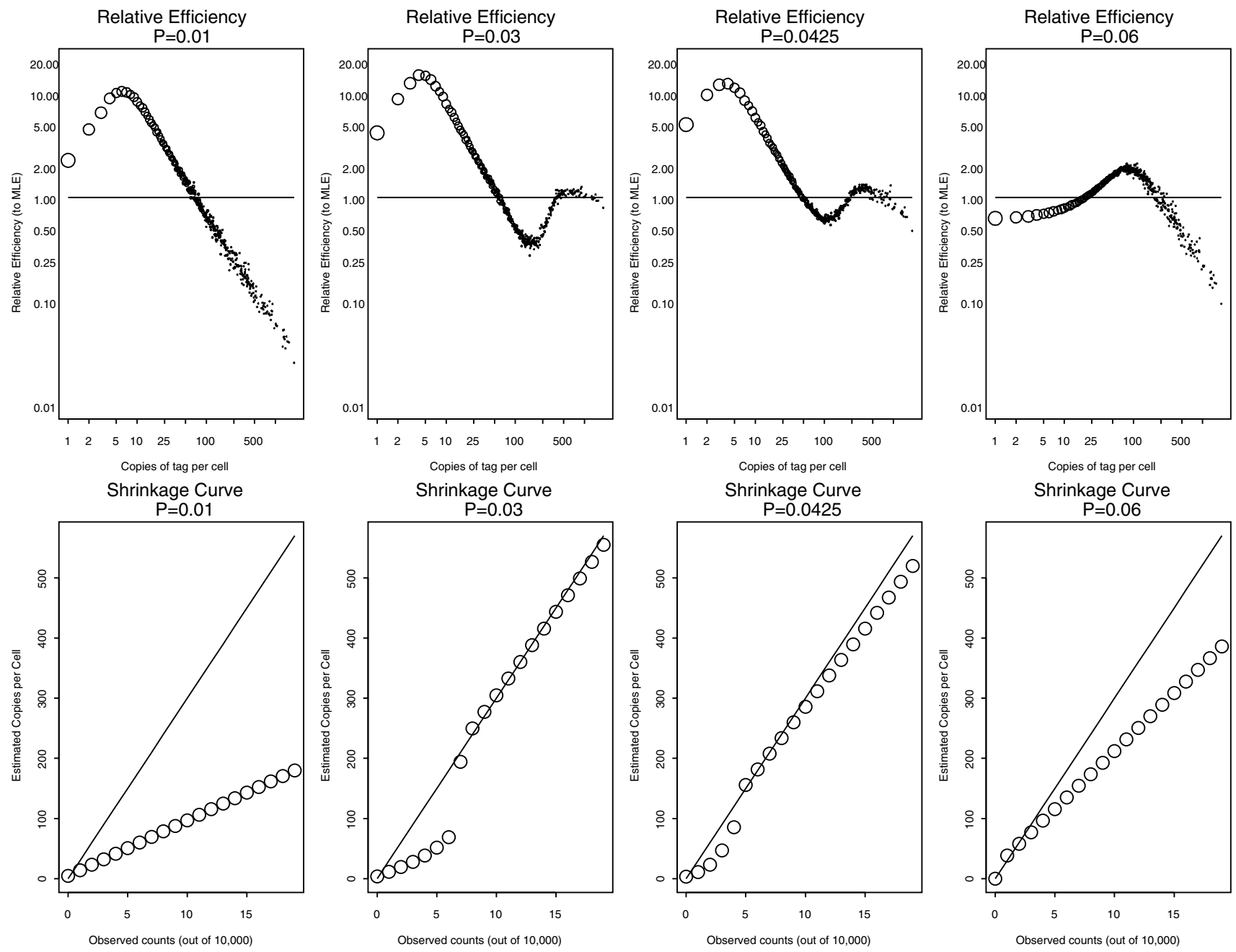

Figure 5. Relative efficiency and shrinkage plots for mixture Dirichlet estimators for various choices of $P$, from a simulation of 100 multinomial samples of size 10,000 taken from a SAGE-like population, with $\theta_{S}=1.0$ and $\theta_{A}=0.5$. Note that the values of $P$ giving a nondegenerate mixture lie within an intermediate range.

converges to a state where all tags are scarce, i.e., the symmetric Dirichlet case. If chosen too large, it converges to a state where all observed tags are abundant. In both of these degenerate cases, the method has a linear shrinkage profile and performs poorly in simulation. Within the nondegenerate region between these two extremes, there is a trade-off in performance between the intermediate and most abundant regions. If $P$ is smaller, there is less shrinkage for the most abundant tags, but a less smooth shrinkage profile, trading off performance in the intermediate range. For larger $P$, there is more shrinkage and thus worse performance for the most abundant tags, but the shrinkage profile is smoother, improving performance in the intermediate range.

When the value of $k$ was misspecified, the mixture method showed similar efficiency gains over the MLE to when it was correctly specified (IMSE $=687$ for $k=25,000$ and IMSE $=$ 651 for $k=65,000$ ), with improvements and trade-offs at the same regions of the parameter space (see Figure 6). This suggests that our method is robust to selection of $k$, as long as $P$ is selected to yield nondegenerate shrinkage profiles.
For $n=50,000$, the mixture Dirichlet again had smaller IMSE than the MLE (173 vs. 201), outperforming the MLE for scarce tags ( $0-15$ copies per cell, $93.5 \%$ of total), with RE of up to 12; it had less efficiency than the MLE for an intermediate range (15-50 copies per cell, $4.9 \%$ of total), and equivalent for the abundant tags. The magnitude of improvement for scarce tags was again larger than the efficiency loss in the intermediate range $(\mathrm{RE}>0.50)$. Turing's estimator had a slightly smaller IMSE (160) than the mixture Dirichlet, and again had extended regions of improved efficiency (0-20 copies per cell) and reduced efficiency (20-150 copies per cell) within the parameter space.

\section{Discussion}

We have introduced a new method for estimating the relative abundance profiles of SAGE tags that explicitly takes into account the skewed nature of the data and so can have efficiency advantages over the MLE. Its key benefit is that the nonlinear shrinkage profile imposed by our prior helps correct for some of the sampling limitations in the data. Other methods 

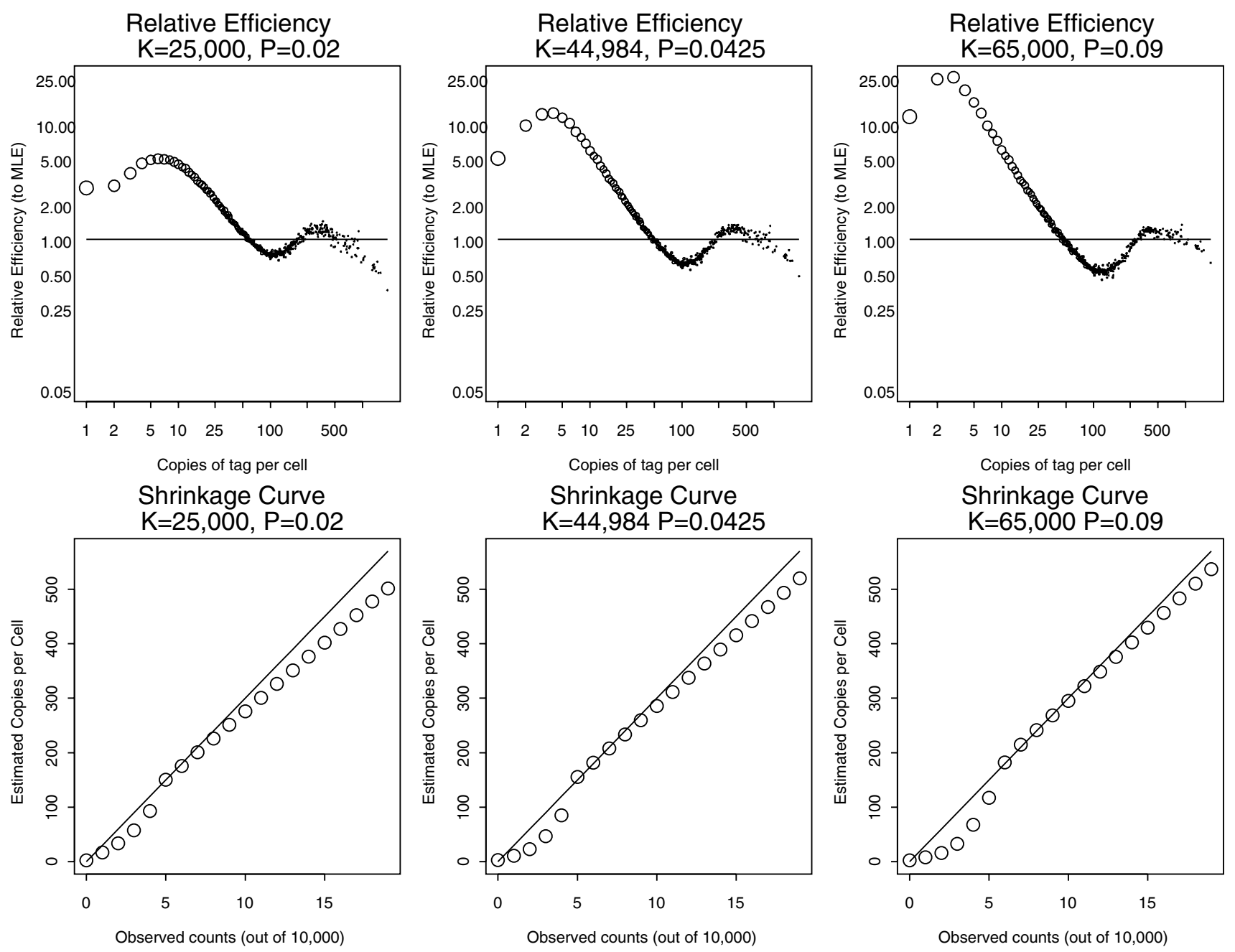

Figure 6. Relative efficiency and shrinkage plots for mixture Dirichlet estimators for various choices of $k$ and $P$ from a simulation of 100 multinomial samples of size 10,000 taken from a SAGE-like population. Note that the performance of the method is robust to misspecification of $k$, given $P$ is chosen to give a nondegenerate mixture.

could be constructed that yield nonlinear shrinkage profiles, and would likely experience similar types of efficiency gains (and trade-offs) to our method.

Turing's estimator, cited in Good (1953), had similar performance to ours, although it is not model based. Blades et al. (2003, to appear) propose a mixture model for SAGE data that partitions the data into scarce and abundant tags based on whether they belong to the linear or noise portions of a $\log$ (number of tags) vs. a $\log$ (frequency of tags) plot. The nonlinear shrinkage profile inherent in their method results in more shrinkage for intermediate and abundant tags than ours, with shrinkage for tags with observed counts into the 10 's and 100's.

The fact that our method flows naturally from a fully specified coherent probability model gives it several inferential advantages over other more ad hoc methods. First, we obtain posterior samples from the joint distribution of all tags' expression levels, from which any inference, univariate or multivariate, can be obtained. For example, estimates, posterior intervals, density estimates, and Bayesian hypothesis tests are available for any quantities derivable from the relative frequencies for any set of tags. Since our model is multivariate, we can also estimate and make probability statements on intertag summaries, e.g., correlations and clustering, which are not available from tag-by-tag modeling or ad hoc approaches.

Second, the model can be extended, if desired, to incorporate other features of SAGE data. For example, in principle, the model can be supplemented to simultaneously estimate $k$, the true number of unique tags in the tissue. Denoising could be incorporated by adding an element onto the model that detects likely sequencing errors. Further, it is possible to add an additional hierarchical level to the model accommodating multiple libraries, so that both the library-to-library and multinomial sources of variability are appropriately taken into account. These extensions require additional work beyond the scope of this article, but are made possible by our model-based approach.

As previously mentioned, the goal of many SAGE experiments is the identification of tags differentially expressed 
between two types of tissue. There are a number of methods in the current literature of testing for differential expression of SAGE tags between two libraries, most of which are applied on a tag-by-tag basis and implicitly involve estimation of $\pi_{1}$ and $\pi_{2}$, the true relative frequencies for a given gene in the two libraries, given the observed counts $A$ and $B$. Zhang et al. (1997) use a permutation test, and Madden et al. (1997) perform a hypothesis test on $\mathrm{H}_{0}: \pi_{1}-\pi_{2}=0$ using the normal approximation to the Poisson. Audic and Claverie (1997) compute the predictive distribution of $B \mid A$ assuming a PoissonGamma model. These methods implicitly use the MLE for $\pi_{1}$ and $\pi_{2}$.

Given two libraries, our method can be applied separately to the two libraries and the posterior samples used to assess differential expression. For example, posterior credible intervals can be constructed on the fold change for each unique tag, i.e., the ratio of the relative abundances, or the posterior probability of a greater than twofold change can be computed, e.g.. If a zero count is observed for one of the two libraries, we can use the posterior samples from one of the anonymous zero count tags. This is reasonable when we are willing to believe that the corresponding mRNA transcript is one of those present in a small quantity in the other tissue, but missed by SAGE due to sampling error. This is an advantage for our method, since other methods must resort to ad hoc adjustments, such as substituting a count of one when zero counts are encountered (e.g., Zhang et al., 1997).

Our simulation results in Section 5 suggest that the mixture Dirichlet estimator has large efficiency benefits over the MLE for the majority of tags that are scarce, and trade-offs of a smaller magnitude for tags in a particular intermediate range and for the most abundant tags. Efficiency gains in estimation of the relative abundances should transfer over to more sensitivity and specificity in assessing differential expression. Thus, we expect to see gains in comparisons that involve at least one scarce tag, and a degree of trade-off when a tag is in the specific regions where the $\mathrm{RE}$ is less than one.

A key question to consider with any shrinkage estimator is whether the gains in efficiency in one part of the parameter space justify the trade-offs in other regions. The answer to this question depends on the investigator, but an argument could be made that the benefits of our method outweigh the costs. First, the region of benefit contains a vast majority of the tags. In most experiments, not all tags are equally important, but frequently the tags that appear to be candidates for differential expression contain a scarce tag in one of the two libraries. Second, the loss of efficiency in the intermediate and most abundant regions is small in magnitude compared to the gain for the scarce tags. Third, we have found that for more abundant tags, the library-to-library variability dominates sampling variability, while for the scarce tags, the sampling variability dominates (Baggerly et al., 2003, to appear). This highlights the benefit of large improvements in the sampling variability of scarce tags, in spite of some efficiency trade-off for more abundant ones. This is especially true considering that low efficiency for the scarce tags is a major factor that drives investigators to use large library sizes at the expense of building fewer libraries. Improved efficiency for the scarce tags has even more clear benefits for other questions that can be addressed with SAGE data, e.g., estimating the number of distinct tags in the tissue and estimating the density of relative expression levels.

We have found that our mixture Dirichlet model is sensitive to choice of $P$. Care must be taken to avoid degenerate shrinkage curves, so we have proposed an automatic diagnostic procedure in Section 3.4.

If interest is mainly in point estimation, one easily implemented alternative to fitting our full model is to apply a suitably nonlinear shrinkage curve to the raw counts to obtain revised counts. Our method must work hand-in-hand with a method for dealing with sequencing errors and estimating the number of unique tags $k$. In multilibrary settings, it is important to also model library-to-library heterogeneity in the relative frequencies. There is clearly more work to be done, but we believe the ideas introduced in this article can serve as an important starting point for a rigorous probability modelbased approach for the analysis of SAGE data.

\section{ACKNOWLEDGEMENTS}

This work was partially supported by National Institutes of Health-The Nuclear Control Institute grant 1 U19 CA849781A1. We thank Natalie Blades for introducing us to the log-log plots for clearly displaying the distribution of true frequencies for SAGE tags, and Peter Müller and Neby Bekele for stimulating discussion. We also thank the associate editor and two referees, whose insightful comments have led to a considerably improved article.

\section{RÉSUMÉ}

SAGE est une technique permettant de quantifier l'expression d'un gène dans un tissu biologique. Cette technique aboutit à l'obtention de données de comptage modélisables par une loi multinomiale possédant les deux caractéristiques suivantes: asymétrie de la distribution des fréquences relatives, et échantillon de petite taille (par rapport à la dimension de la loi multinomiale). En raison de ces caractéristiques, un échantillon SAGE peut manquer un grand nombre de types d'ARNm pourtant présents dans le tissu. Dès lors qu'ils ignorent ce problème des types manquants, des estimateurs empiriques de la fréquence relative des types d'ARNm tendent naturellement à surestimer la fréquence des types rares trouvés au moins une fois, lesquels forment la grande majorité des types observés. Nous avons donc développé une nouvelle procédure d'estimation bayésienne comportant une information a priori tenant compte des caractéristiques précitées. Cette procédure aboutit à des estimateurs non linéaires "à rétrécisseur" (shrinkage) plus efficaces que les estimateurs du maximum de vraisemblance. La distribution a priori est constituée d'un mélange de lois de Dirichlet, les types ARNm étant stochastiquement classés en types "rares" et "abondants" - une distribution multivariée a priori est calculée séparément pour chacune des deux classes. Différentes simulations montrent que, sur les scénarios SAGE envisagés, cet estimateur a un meilleur IMSE (somme des écarts quadratiques moyens des erreurs sur la fréquence, cumulée sur l'ensemble des types) que l'estimateur du maximum de vraisemblance, c'est-à-dire qu'il propose, pour tous les échantillons simulés, des profils de fréquences relatives plus proches de la réalité, au sens de la distance euclidienne. Nous appliquons cette méthode à une bibliothèque SAGE de tissus issus de côlons normaux, et discutons de ses implications quant à l'évaluation de l'expression différentielle. 


\section{REFERENCES}

Audic, S. and Claverie, J. M. (1997). The significance of digital gene expression profiles. Genome Research 7, 986995.

Baggerly, K. A., Deng, L., Morris, J. S., and Aldez, C. M. (2003). Differential expression in SAGE: Accounting for normal between-library variation. Bioinformatics, to appear.

Blades, N. (2002). Noise and shadows, statistical issues with SAGE data. Ph.D. thesis, Johns Hopkins University, Baltimore.

Blades, N., Kern, S., Jones, J., and Parmigiani, G. (2003). Denoising SAGE libraries: A mixture model approach. Bioinformatics, to appear.

Bunge, J. and Fitzpatrick, M. (1993). Estimating the number of species: A review. Journal of the American Statistical Association 88, 364-373.

Colinge, J. and Feger, G. (2001). Detecting the impact of sequencing errors on SAGE data. Bioinformatics 17(9), 840-842.

George, E. I. (1986). Minimax multiple shrinkage estimation. Annals of Statistics 14, 188-205.

Good, I. J. (1953). The population frequencies of species and the estimation of population parameters. Biometrika $\mathbf{4 0}$, 237-264.

Gruber, M. H. J. (1998). Improving Efficiency by Shrinkage. New York: Marcel Dekker.

Hastie, N. D. and Bishop, J. O. (1976). The expression of three abundance classes of messenger RNA in mouse tissues. Cell 9, 761-774.
James, W. and Stein, C. (1961). Estimation with quadratic loss. In Proceedings of the Fourth Berkeley Symposium on Mathematical Statistics and Probability, Volume 1, 361379. Berkeley: University of California Press.

Jeffreys, H. (1961). Theory of Probability, 3rd edition. Oxford: Clarendon Press.

Kuznetsov, V. A. (2001). Distribution associated with stochastic processes of gene expression in a single eukaryotic cell. EURASIP Journal on Applied Signal Processing 4, 285-296.

Madden, S. L., Galella, E. A., Zhu, J., Bertelsen, A. H., and Beaudry, G. A. (1997). SAGE transcript profiles for p53dependent growth regulation. Oncogene 5, 1079-1085.

Polyak, K. and Riggins, G. J. (2001). Gene discovery using the serial analysis of gene expression technique: Implications for cancer research. Journal of Clinical Oncology 19, 2948-2958.

Stollberg, J., Urschitz, J., Urban, Z., and Boyd, C. D. (2000). A quantitative evaluation of SAGE. Genome Research 10, 1241-1248.

Velculescu, V. E., Madden, S. L., Zhang, L., et al. (1999). Analysis of human transcriptomes. Nature Genetics 23, 387-388.

Zhang, L., Zhou, W., Velculescu, V. E., Kern, S. E., Hruban, R. H., Hamilton, S. R., Vogelstein, B., and Kinzler, K. W. (1997). Gene expression profiles in normal and cancer cells. Science 276, 1268-1272.

\section{Received May 2002. Revised January 2003.} Accepted January 2003. 